\title{
Gankyrin is essential for hypoxia enhanced metastatic potential in breast cancer cells
}

\author{
LIUCUN GAO $^{1 *}$, HUAHONG XIE $^{2 *}$, LIHOU DONG $^{1 *}$, JIA ZOU $^{1}, \mathrm{JIE} \mathrm{FU}^{1}$, \\ XIN GAO ${ }^{1}$, LUN OU ${ }^{1}$, SHENSI XIANG ${ }^{1}$ and HAIFENG SONG ${ }^{1}$ \\ ${ }^{1}$ Department of Pharmacology and Toxicology, Beijing Institute of Radiation Medicine, Beijing; \\ ${ }^{2}$ State Key Laboratory of Cancer Biology, Xijing Hospital of Digestive Diseases, Fourth Military Medical University, \\ Xi'an, Shaanxi, P.R. China
}

Received July 1, 2013; Accepted December 2, 2013

DOI: $10.3892 / \mathrm{mmr} .2013 .1860$

\begin{abstract}
Hypoxia, a critical regulator of tumor growth and metastasis, induces the transcriptional activation of several pathways involved in proliferation, migration and invasion. Gankyrin was found to be overexpressed, and also promoted the metastasis in breast cancer cells, which is also involved in the regulation of hypoxia inducible factor-1 and hypoxia-inducible factor- $1 \alpha$. The present study showed that gankyrin mRNA and protein expression were increased under hypoxic conditions in the BT474 breast cancer cell line, accompanied with increased ability of cell migration and invasion. Lentivirus-mediated siRNA targeting gankyrin was transfected into BT474 cells. Wound-healing and transwell experiments showed that gankyrin deletion abrogated the increased migration and invasion of BT474 cells due to hypoxia. In addition, E-cadherin was found to be involved in the gankyrin induced invasion of breast cancer cells due to hypoxia. The present study indicated that gankyrin deletion abrogated the increased metastatic potential of breast cancer cells under hypoxic conditions partly through regulating E-cadherin, suggesting that an improved understanding of gankyrin may offer a potential therapeutic target for the treatment of human breast cancer metastasis.
\end{abstract}

\section{Introduction}

In females, breast cancer is the most frequently diagnosed type of cancer and the leading cause of cancer-related mortality worldwide (1). Approximately half of the breast cancer patients and $60 \%$ of the mortalities were presented

Correspondence to: Professor Haifeng Song, Department of Pharmacology and Toxicology, Beijing Institute of Radiation Medicine, 27 Taiping Road, Beijing 100850, P.R. China

E-mail: songhf@nic.bmi.ac.cn

${ }^{*}$ Contibuted equally

Key words: gankyrin, breast cancer, hypoxia, E-cadherin in economically developing countries in 2008 (1). Cancers characteristically develop rapidly in primary and metastatic locations, accompanied by novel blood vessel development and poor blood flow, resulting in a progressively hypoxic and hypoglycemic microenvironment for cancer cells $(2,3)$. Hypoxia is identified as a physiological abnormality in solid tumors and is involved in the malignant progression of a number of cancers (4).

Although hypoxia is toxic to cancer and normal cells, cancer cells undergo genetic and adaptive changes that allow them to survive and metastasize in a hypoxic environment. These processes contribute to the malignant phenotype and to aggressive tumor behavior. In solid tumors, hypoxia can promote malignant growth, and confer resistance to chemotherapy and metastasis by altering gene expression, particularly in breast cancer (5). However, the mechanisms of hypoxia-induced tumor metastasis in breast cancer requires further clarification.

Gankyrin, also known as p28GANK and PSMD10, is a $25 \mathrm{KDa}$ protein with 226 amino acids, which is comprised of seven ankyrin repeats (6). Gankyrin has been confirmed as a bridging factor between the proteasome and various tumor-associated substrates, including $\mathrm{pRb}$ and p53 (7). Gankyrin increases the hyperphosphorylation of $\mathrm{Rb}$ by activating CDK4 and therefore activates E2F-dependent transcription of DNA synthesis genes. It can also activate the ubiquitin protein ligase murine double minute 2 and lead to the proteasomal degradation of p53 (8). Previous studies showed that gankyrin was overexpressed in various human cancers, including hepatocellular carcinoma (9) and esophageal squamous cell (10), colorectal (11), pancreatic (12) and oral (13) cancer. In breast cancer, gankyrin is frequently overexpressed and is associated with ErbB2 expression (14), which also promotes breast cancer cell metastasis by regulating Rac1 activity (15). Previously, a study found that gankyrin may bind to and sequester factor inhibiting hypoxia inducible factor-1 (FIH-1), resulting in a decreased interaction between FIH-1 and hypoxia-inducible factor- $1 \alpha(\mathrm{HIF}-1 \alpha)$, which increased the activity of HIF-1 to promote vascular endothelial growth factor (VEGF) production (16). Therefore, gankyrin may be significant in the hypoxia-induced malignant progression of human cancer. 
In the present study, the capability of hypoxia to increase gankyrin mRNA and protein expression in breast cancer cell lines, and the roles of gankyrin in the hypoxia induced invasion and metastasis of breast cancer cells was investigated using lentivirus-mediated siRNA targeting gankyrin.

\section{Materials and methods}

Cell lines and tissue samples. BT474 and MCF7 human breast cancer cell lines were provided by colleagues in the Department of Pharmacology and Toxicology, Beijing Institute of Radiation Medicine (Beijing, China), and cell lines were routinely cultured in RPMI 1640 medium containing 10\% fetal calf serum. For the hypoxic condition, cells were cultured in a modular incubator chamber (Billups-Rothenberg, San Diego, CA, USA), which was provided with $1 \% \mathrm{O}_{2}, 5 \% \mathrm{CO}_{2}$ and $94 \%$ $\mathrm{N}_{2}$, and for normoxic conditions, cells were maintained under $\left(20 \% \mathrm{O}_{2}, 5 \% \mathrm{CO}_{2}\right.$, and $\left.75 \% \mathrm{~N}_{2}\right)$.

Quantitative polymerase chain reaction ( $q P C R)$. Total RNA of breast cancer cell lines in normal conditions and undergoing hypoxia treatment for $0,12,24$ and $36 \mathrm{~h}$ were extracted using TRIzol (Invitrogen Life Technologies, Carlsbad, CA, USA) according to the manufacturer's instructions. The primer sequences used were as follows: Forward: 5'-TCTTCAAGCCATCCTGTGTG-3' and reverse: 5'-TGGTGATGTTGGACTCCTCA-3' for gankyrin; and forward: 5'-ATGATATCGCCGCGCTCGTC-3' and reverse: 5'-CGCTCGGTGAGGATCTTCA-3' for $\beta$-actin. The qPCR assays were performed and results were calculated as previously described (13).

Western blotting. Total protein of breast cancer cell lines under normal conditions and hypoxia treatment for $0,12,24$ and $36 \mathrm{~h}$ were extracted following a previous study (11). The total protein was separated on a $12 \%$ (for gankyrin and $\beta$-actin) or $8 \%$ (for E-cadherin) polyacrylamide gels, and electrotransferred on to a nitrocellulose membrane. Mouse polyclonal anti-gankyrin (Santa Cruz Biotechnology Inc., Santa Cruz, CA, USA; 1:100), mouse monoclonal anti-E-cadherin (Santa Cruz Biotechnology Inc.; 1:100), and mouse monoclonal anti-beta actin (Sigma, St. Louis, MO, USA; 1:3,000).

Lentivirus-mediated siRNA construction and transfection. The lentivirus-mediated siRNA targeting gankyrin was subcloned into the PGC-LV system with enhanced green fluorescent protein (EGFP). The siRNA interfering sequence was 5'-CTGACCAGGACAGCAGAAC-3'. The control lentivirus was also enhanced with EGFP. The siRNA and control lentivirus were transfected into BT474 cells, and the GFP-positive cells were purified by flow cytometry (FACScan; Becton Dickinson, San Jose, CA, USA), and labeled si- and con-BT474.

Wound-healing experiment. The wound-healing assay was used to detect the migration of cells as described previously (17). Briefly, $2 \times 10^{6}$ cells of each cell line were plated in a 60-mm-diameter dish, and cultured until the cells reached confluency. A plastic pipette tip was then drawn across the center of the plate to produce clean 1-mm-wide wound areas.
Following $48 \mathrm{~h}$ culturing in normoxia or hypoxic conditions, a phase-contrast microscope (Olympus, Tokyo, Japan) was used to detect the cells in the wound areas.

Transwell assays. Cell migration and invasion assays were performed using transwells (8- $\mu 1$ pore size; Corning Inc, Acton, MA, USA). For transwell migration assays, $1 \times 10^{5}$ cells were plated in the top chamber lined with a non-coated membrane. For invasion assays, the chamber inserts were coated at a concentration of $200 \mathrm{mg} / \mathrm{ml}$ in Matrigel (BD Biosciences, San Jose, CA, USA) and dried under sterile conditions for $10 \mathrm{~h}$. Cells were prepared at concentration of $1 \times 10^{5}$ cells in RPMI 1640 culture solution without fetal bovine serum or growth factors, and $200 \mu \mathrm{l}$ mixed liquor was plated into the top chamber. Next, $400 \mu \mathrm{l}$ medium supplemented with $20 \%$ fetal bovine serum was plated into the lower chamber. Following incubation in normal culture conditions for $24 \mathrm{~h}$, cells in the top chambers were wiped to remove the non-invasive cells, and invaded cells on the underside membrane were incubated with $10 \%$ paraformaldehyde for $10 \mathrm{~min}$ and stained in $0.1 \%$ crystal violet. Following three washes with phosphate-buffered saline and air-drying, cells were counted by phase-contrast microscopy at magnification, x200 on 10 random visual fields in each well. Each experimental condition was repeated in triplicate.

Statistical analysis. The SPSS 17.0 software (SPSS Inc., Chicago, IL, USA) was used to evaluate the statistical differences, and $\mathrm{P}<0.05$ was considered to indicate a statistically significant difference. A t-test was performed to analyze the difference between two groups.

\section{Results}

Expression of gankyrin in breast cancer cell lines. qPCR and western blot analysis were used to detect the expression of gankyrin in MCF7 and BT474 breast cancer cell lines under normoxic conditions. The mRNA of gankyrin expression was markedly higher in MCF7 compared with BT474 cells (Fig. 1A). Consistent with mRNA expression, the gankyrin protein expression was markedly higher in MCF7 compared with BT474 cells (Fig. 1B). Therefore, the BT474 cell line was used for further investigation under hypoxic conditions.

Hypoxia induces overexpression of gankyrin in BT474 cells. Under normoxic conditions, low expression of gankyrin was detected in BT474 cells. Following induction of hypoxia for $0,12,24$ and $36 \mathrm{~h}$, the mRNA and protein were collected for qPCR and western blot analyses. As shown in Fig. 2A, gankyrin mRNA expression exhibited a marked overexpression tendency under hypoxic conditions in BT474 cells. Consistent with results of qPCR, western blot analysis showed a gradual increase in gankyrin expression under hypoxic conditions (Fig. 2B), indicating that hypoxia induced the overexpression of gankyrin in BT474 cells.

Hypoxia increases the migration and invasion of BT474 cells. Wound-healing assays were performed to compare the migration rate of the BT474 cells under normoxic and hypoxic conditions. As shown in Fig. 3A, hypoxia markedly increased 
$\mathbf{A}$

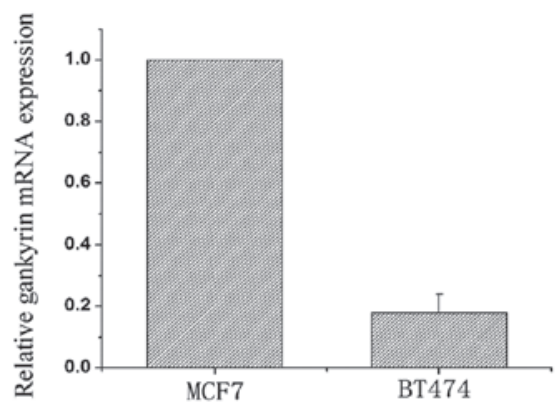

B

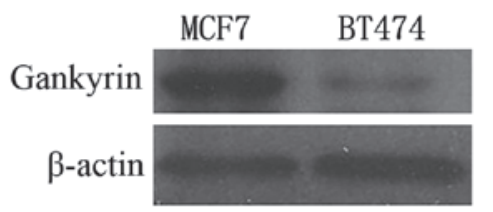

Figure 1. Gankyrin expression in breast cancer cell lines under normoxia. (A) qPCR was performed to detect the mRNA expression of gankyrin in MCF7 and BT474 cells. (B) Western blot analysis was used to detect gankyrin protein expression in MCF7 and BT474 cells. $\beta$-actin was used as a loading control. qPCR, quantitative polymerase chain reaction.

A

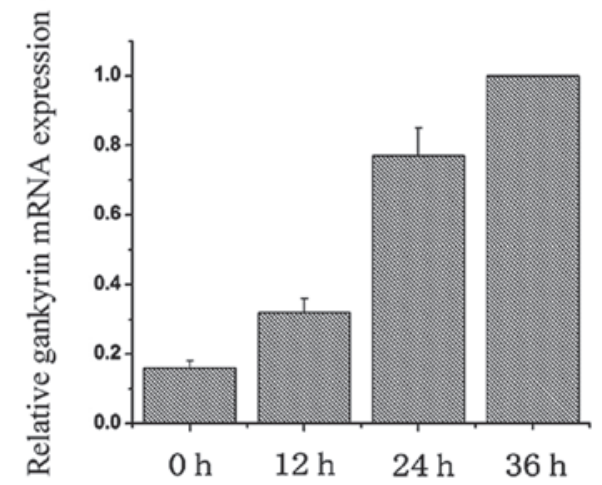

B

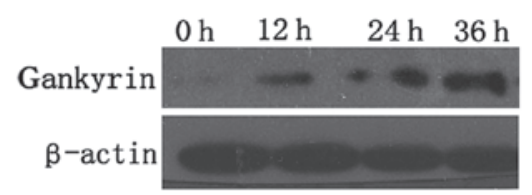

Figure 2. Hypoxia induced overexpression of gankyrin in BT474 cells. (A) qPCR was performed to detect the mRNA expression of gankyrin in BT474 cells under hypoxic conditions. (B) Western blot analysis was used to detect the gankyrin protein expression in BT474 cells under hypoxic conditions. $\beta$-actin was used as a loading control. qPCR, quantitative polymerase chain reaction.

BT474 cell migration from the edge of the wound compared with normoxic conditions (Fig. 3A). Next, transwell assays were used to detect the migration and invasion of BT474 cells. Following culturing in normoxic or hypoxic conditions for $36 \mathrm{~h}$, hypoxia markedly increased the migration and invasion ability of BT474 cells compared with normoxia (Fig. 3B and $\mathrm{C}$ ). These data indicated that hypoxia increased the migration and invasion of BT474 cells.
A

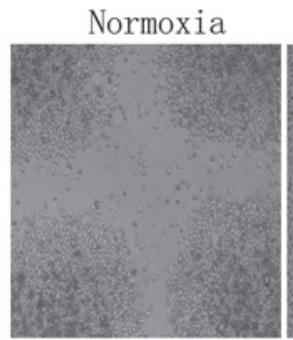

B

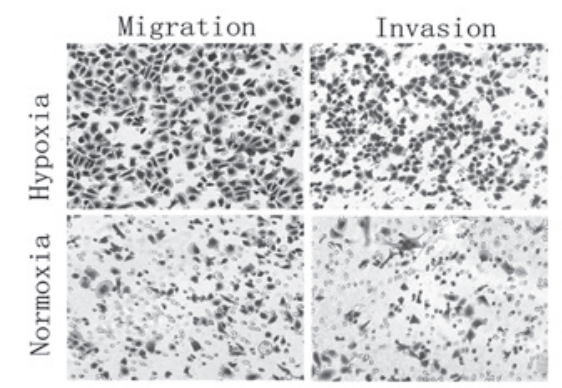

C

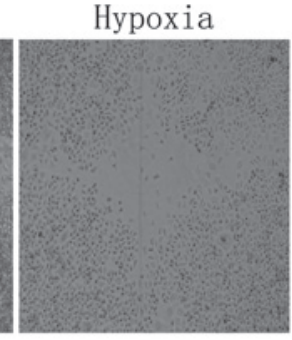

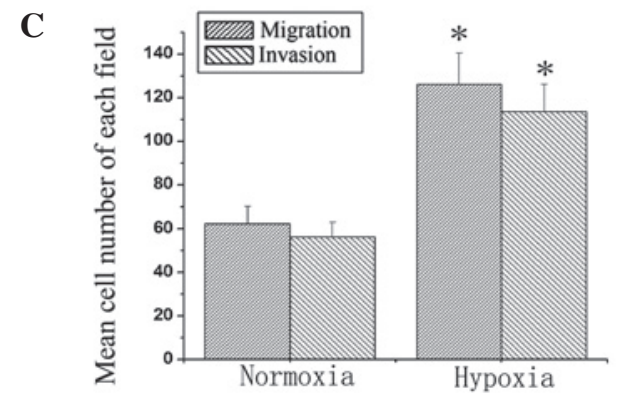

Figure 3. Hypoxia increased the migration and invasion of BT474 cells. (A) Wound-healing assays showed that hypoxia increased the migration of BT474 cells. (B) Transwell assays revealed that hypoxia markedly increased the migration and invasion of BT474 cells compared with normoxia. (C) Statistical analyses indicated that the ability of migration and invasion of BT474 cells were significantly higher under hypoxic than normoxic conditions. ${ }^{*} \mathrm{P}<0.05$, vs normoxic conditions.

Gankyrin deletion abrogates the increased metastatic potential of BT474 cells due to hypoxia. To investigate the roles of gankyrin in the hypoxia-induced metastatic potential of BT474 cells, lentivirus-mediated siRNA targeting gankyrin was transfected into BT474 cells. As shown in Fig. 4A, hypoxia could not induce the overexpression of gankyrin in BT474 cells transfected with siRNA compared with normoxic conditions (Fig. 4B). Wound-healing (Fig. 4C) and transwell assays assays (Fig. 4D and E) showed that the migration and invasion of gankyrin siRNA-transfected cells were significantly inhibited compared with control siRNA transfected cells. These findings suggest that gankyrin deletion abrogates the increased metastatic potential of BT474 cells due to hypoxia.

E-cadherin is involved in the gankyrin-induced invasion of breast cancer cells due to hypoxia. A previous study showed that E-cadherin expression was markedly inhibited under hypoxic conditions in breast cancer cells (18). In addition, gankyrin was reported to be involved in the metastasis of hepatocellular carcinoma invasiveness and metastasis (19). Therefore, the role of E-cadherin in the gankyrin-induced invasion of breast cancer cells due to hypoxia was investigated. Western blotting showed that E-cadherin expression 
A
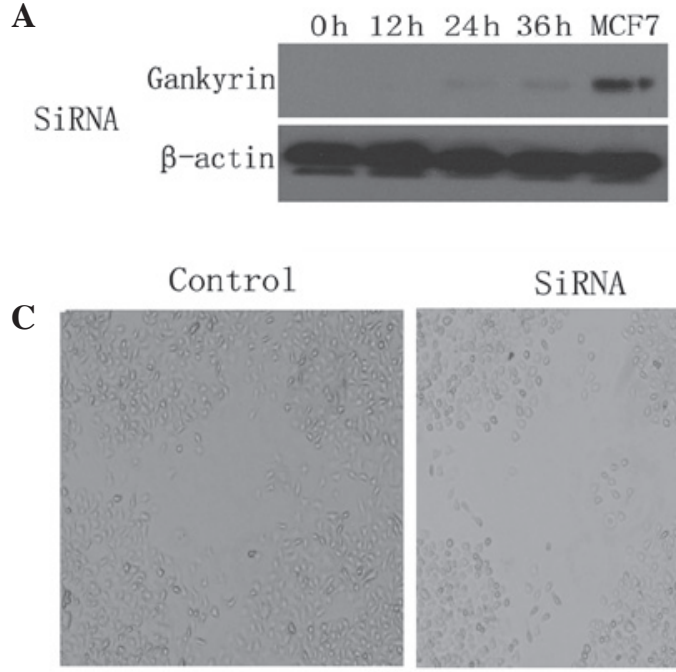

SiRNA

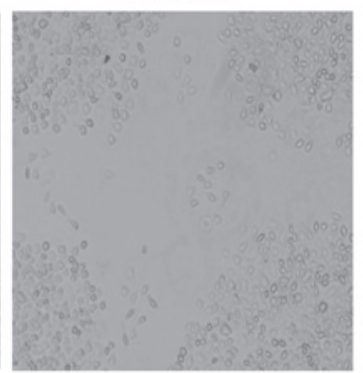

B
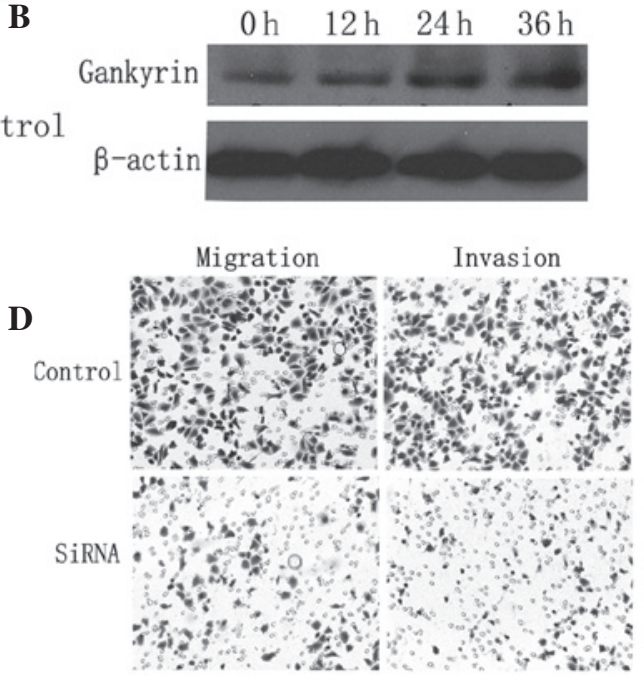

$\mathbf{E}$

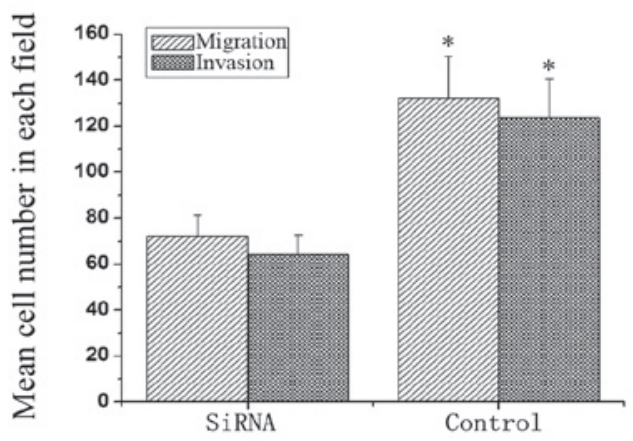

Figure 4. Gankyrin deletion abrogated the increased metastatic potential of BT474 cells due to hypoxia. (A and B) Western blot analysis was used to detect gankyrin protein expression in si-BT474 (siRNA) and con-BT474 (control) cells under hypoxic conditions. MCF7 cells were used as a positive control, and $\beta$-actin was used as a loading control. (C) Wound-healing assays showed markedly decreased migration of si-BT474 cells compared with con-BT474. (D and E) Transwell assays revealed that the migration and invasion of si-BT474 cells was significantly decreased compared with con-BT474 under hypoxic conditions. ${ }^{*} \mathrm{P}<0.05$, vs. con-BT474 cells.

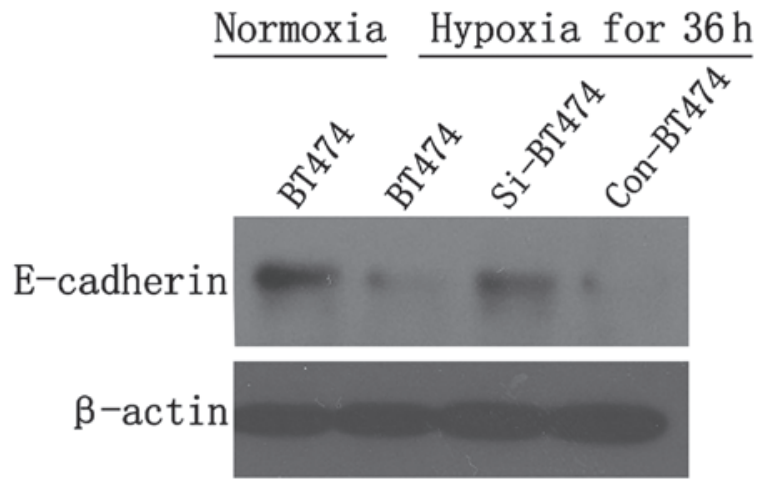

Figure 5. Gankyrin deletion could abrogate the inhibition of E-cadherin expression in BT474 cells due to hypoxia. $\beta$-actin was used as a loading control.

was markedly decreased following culturing for $36 \mathrm{~h}$ under hypoxic conditions in BT474 cells compared with normoxic conditions. Under hypoxic conditions, the expression of E-cadherin was markedly higher in si-BT474 cells compared with con-BT474 cells (Fig. 5), indicating that hypoxia may inhibit the expression of E-cadherin, and gankyrin deletions may abrogate the inhibition of E-cadherin expression in BT474 cells due to hypoxia.

\section{Discussion}

In breast cancer, hypoxia is a critical regulator for the transcriptional activation of several pathways, including angiogenesis, immortalization, invasion and metastasis (5). A previous study showed that HIF-1 $\alpha$ and HIF-2 $\alpha$ accumulated in breast cancer cells with hypoxia and potentiated Notch signaling (20). The hypoxic conditions increased the expression of BlyS in human breast cancer cell lines, and upregulation of BlyS led to activation and nuclear translocation of NF- $\mathrm{kB}$ p65, which also increased the migration and invasion of breast cancer cells (21). A recent study showed that gankyrin binds to and sequesters factors inhibiting FIH-1, resulting in a decreased interaction between FIH-1 and HIF-1 $\alpha$, which increased activity of HIF-1 to promote VEGF production (16), indicating that gankyrin is significant in the hypoxia-associated phenotype in breast cancer cells. However, the role and mechanism of gankyrin in hypoxic environments remain unknown.

In the present study, the mRNA and protein of gankyrin were observed to be increased in breast cancer cells with hypoxia, accompanied with the increased migration and invasion in BT474 cells. To investigate the roles of gankyrin in the hypoxia-induced metastatic potential, lentiviral-mediated siRNA targeting gankyrin was transfected into BT474 cells. Compared with control siRNA transfection, the expression 
of gankyrin did not increase under hypoxic conditions in gankyrin siRNA transfected cells, indicating that siRNA is capable of inhibiting the increased gankyrin expression due to hypoxia in BT474 cells. Wound-healing and transwell assays confirmed that gankyrin deletion abrogated the increased metastatic potential of BT474 cells due to hypoxia. These findings indicated enhanced breast cancer cell migration in response to gankyrin under hypoxic conditions, which may be a potential therapeutic target for breast cancer metastasis treatment. According to a previous report, gankyrin expression may be modulated by growth factors, including epidermal growth factor or hepatocyte growth factor stimulation, and Ras activation through the activation of phosphoinositide 3-kinase signaling (22). The present study showed that hypoxia is another factor that results in stimulation of gankyrin expression.

Epithelial-to-mesenchymal transition is induced by the loss of cell adhesion, repression of E-cadherin expression and increased cell migration and invasion. E-cadherin expression was repressed during the metastasis of breast cancer. A previous study showed that E-cadherin expression was markedly inhibited under hypoxic conditions in breast cancer cells (18). Overexpression of gankyrin significantly downregulated the expression of E-cadherin, and suppression of gankyrin expression using adenovirus-delivered siRNA markedly increased the expression of E-cadherin in HCC cell lines (19). Therefore, we further investigated the associations between gankyrin and E-cadherin involved in promoting the migration and invasion of breast cancer cells under hypoxic conditions. Consistent with a previous study, western blot analyses showed that E-cadherin expression was decreased under hypoxic conditions (18). However, when gankyrin was deleted by siRNA, the expression of E-cadherin was not markedly altered and no marked metastatic potential change was observed in BT474 cells under hypoxic conditions. This indicated that E-cadherin was involved in the gankyrin-induced invasion of breast cancer cells due to hypoxia.

In addition, to investigate the significance of gankyrin expression in breast cancer tissues, 104 pairs of breast cancer and matched non-tumor tissues were used for immunohistochemical staining. Further analyses revealed that gankyrin expression was associated with a high histological tumor grade, estrogen/progesterone receptors and axillary lymph node status. These data were consistent with previous studies of the significance of gankyrin expression in breast cancer tissues $(14,15)$.

The present study demonstrated that the increased expression of gankyrin was significant in the hypoxia-enhanced metastatic potential in breast cancer cells partly through the regulation of E-cadherin. Further studies are required to determine the mechanism by which hypoxia induces the overexpression of gankyrin in breast cancer cells.

\section{Acknowledgements}

This study was supported by a grant from the National Natural Science Foundation of China (grant no. 30900675).

\section{References}

1. Jemal A, Bray F, Center MM, Ferlay J, Ward E and Forman D: Global cancer statistics. CA Cancer J Clin 61: 69-90, 2011.

2. Onozuka HK, Tsuchihara K and Esumi H: Hypoglycemic/hypoxic condition in vitro mimicking the tumor microenvironment markedly reduced the efficacy of anticancer drugs. Cancer Sci 102: 975-982, 2011.

3. Louie E, Nik S, Chen JS, et al: Identification of a stem-like cell population by exposing metastatic breast cancer cell lines to repetitive cycles of hypoxia and reoxygenation. Breast Cancer Res 12: R94, 2010.

4. Pouysségur J, Dayan F and Mazure NM: Hypoxia signalling in cancer and approaches to enforce tumour regression. Nature 441: 437-443, 2006.

5. Harris AL: Hypoxia - a key regulatory factor in tumour growth. Nat Rev Cancer 2: 38-47, 2002 .

6. Krzywda S, Brzozowski AM, Higashitsuji H, et al: The crystal structure of gankyrin, an oncoprotein found in complexes with cyclin-dependent kinase 4, a $19 \mathrm{~S}$ proteasomal ATPase regulator, and the tumor suppressors Rb and p53. J Biol Chem 279: 1541-1545, 2004.

7. Lozano G and Zambetti GP: Gankyrin: an intriguing name for a novel regulator of p53 and RB. Cancer Cell 8: 3-4, 2005.

8. Higashitsuji $\mathrm{H}$, Higashitsuji $\mathrm{H}$, Itoh $\mathrm{K}$, et al: The oncoprotein gankyrin binds to MDM2/HDM2, enhancing ubiquitylation and degradation of p53. Cancer Cell 8: 75-87, 2005.

9. Fu XY, Wang HY, Tan L, Liu SQ, Cao HF and Wu MC: Overexpression of p28/gankyrin in human hepatocellular carcinoma and its clinical significance. World $\mathrm{J}$ Gastroenterol 8: 638-643, 2002.

10. Ortiz CM, Ito T, Tanaka E, et al: Gankyrin oncoprotein overexpression as a critical factor for tumor growth in human esophageal squamous cell carcinoma and its clinical significance. Int J Cancer 122: 325-332, 2008.

11. Tang S, Yang G, Meng Y, et al: Overexpression of a novel gene gankyrin correlates with the malignant phenotype of colorectal cancer. Cancer Biol Ther 9: 88-95, 2010.

12. Meng Y, He L, Guo X, et al: Gankyrin promotes the proliferation of human pancreatic cancer. Cancer Lett 297: 9-17, 2010.

13. Li J, Knobloch TJ, Kresty LA, et al: Gankyrin, a biomarker for epithelial carcinogenesis, is overexpressed in human oral cancer. Anticancer Res 31: 2683-2692, 2011.

14. Kim YH, Kim JH, Choi YW, et al: Gankyrin is frequently overexpressed in breast cancer and is associated with ErbB2 expression. Exp Mol Pathol 94: 360-365, 2012.

15. Zhen C, Chen L, Zhao Q, et al: Gankyrin promotes breast cancer cell metastasis by regulating Racl activity. Oncogene 32: 3452-3460, 2013.

16. Liu Y, Higashitsuji H, Higashitsuji $\mathrm{H}$, et al: Overexpression of gankyrin in mouse hepatocytes induces hemangioma by suppressing factor inhibiting hypoxia-inducible factor-1 (FIH-1) and activating hypoxia-inducible factor-1. Biochem Biophys Res Commun 432: 22-27, 2013.

17. Liu N, Zhang G, Bi F, et al: RhoC is essential for the metastasis of gastric cancer. J Mol Med (Berl) 85: 1149-1156, 2007.

18. Yang G, Tang SQ, Huang DS, Wang JW, Liu Y and Wang JY: Aplastic anemia transformed into acute myeloblastic leukemia M1 8 years later: a case report. Zhonghua Er Ke Za Zhi 43: 221, 2005 (In Chinese).

19. Fu J, Chen Y, Cao J, et al: p28GANK overexpression accelerates hepatocellular carcinoma invasiveness and metastasis via phosphoinositol 3-kinase/AKT/hypoxia-inducible factor-1alpha pathways. Hepatology 53: 181-192, 2011.

20. Chen J, Imanaka N, Chen J and Griffin JD: Hypoxia potentiates Notch signaling in breast cancer leading to decreased E-cadherin expression and increased cell migration and invasion. $\mathrm{Br} \mathrm{J}$ Cancer 102: 351-360, 2010.

21. Zhu J, Sun L, Lin S, et al: BlyS is up-regulated by hypoxia and promotes migration of human breast cancer cells. J Exp Clin Cancer Res 31: 31, 2012.

22. Dong LW, Yang GZ, Pan YF, et al: The oncoprotein p28GANK establishes a positive feedback loop in beta-catenin signaling. Cell Res 21: 1248-1261, 2011. 